\title{
Persistent Accumulation of Calcium/Calmodulin-Dependent Protein Kinase II in Dendritic Spines after Induction of NMDA Receptor-Dependent Chemical Long-Term Potentiation
}

\author{
Nikolai Otmakhov, ${ }^{1}$ Jung-Hwa Tao-Cheng, ${ }^{3}$ Stephen Carpenter, ${ }^{1}$ Brent Asrican, ${ }^{1}$ Ayse Dosemeci, ${ }^{4}$ Thomas S. Reese, ${ }^{3}$ \\ and John Lisman ${ }^{2}$ \\ ${ }^{1}$ Department of Biology and ${ }^{2}$ Volen Center for Complex Systems, Brandeis University, Waltham, Massachusetts 02454, ${ }^{3}$ National Institute of Neurological \\ Disorders and Stroke, Bethesda, Maryland 20892, and ${ }^{4}$ Marine Biological Laboratory, Woods Hole, Massachusetts 02543
}

\begin{abstract}
Calcium/calmodulin-dependent protein kinase II (CaMKII) is a leading candidate for a synaptic memory molecule because it is persistently activated after long-term potentiation (LTP) induction and because mutations that block this persistent activity prevent LTP and learning. Previous work showed that synaptic stimulation causes a rapidly reversible translocation of CaMKII to the synaptic region. We have now measured green fluorescent protein (GFP)-CaMKII $\alpha$ translocation into synaptic spines during NMDA receptor-dependent chemical LTP (cLTP) and find that under these conditions, translocation is persistent. Using red fluorescent protein as a cell morphology marker, we found that there are two components of the persistent accumulation. cLTP produces a persistent increase in spine volume, and some of the increase in GFP-CaMKII $\alpha$ is secondary to this volume change. In addition, cLTP results in a dramatic increase in the bound fraction of GFP-CaMKII $\alpha$ in spines. To further study the bound pool, immunogold electron microscopy was used to measure CaMKII $\alpha$ in the postsynaptic density (PSD), an important regulator of synaptic function. cLTP produced a persistent increase in the PSD-associated pool of CaMKII $\alpha$. These results are consistent with the hypothesis that CaMKII $\alpha$ accumulation at synapses is a memory trace of past synaptic activity.
\end{abstract}

Key words: protein kinase; postsynaptic density; imaging; synapse; LTP; long-term potentiation; EM; tissue culture; fluorescence

\section{Introduction}

Substantial progress is being made in understanding the biochemical events that underlie synaptic long-term potentiation (LTP). The process is initiated by $\mathrm{Ca}^{2+}$ entry through NMDA channels (Bliss and Collingridge, 1993). The resulting activation of calcium/calmodulin-dependent protein kinase II (CaMKII) (Fukunaga et al., 1993; Barria et al., 1997; Ouyang et al., 1999) is a critical next step in the process (Malinow et al., 1989; Silva et al., 1992). Indeed, mutation of a single site on CaMKII that prevents its persistent activation (T286A) blocks LTP and strongly interferes with memory in the Morris water maze (Giese et al., 1998). Because of the importance of CaMKII in synaptic memory (for

Received Sept. 8, 2003; revised Aug. 19, 2004; accepted Aug. 24, 2004.

This work was supported by Grant R01 NS-27337 from the National Institutes of Health/National Institute of Neurological Disorders and Stroke. We thank Tobias Meyer and Neal Waxham for GFP-CaMKII constructs, members of the Leslie Griffith laboratory for help with DNA amplification, Ken Sugino for initial assistance with slice culture, Karra Pratt for introducing us to gene gun transfection, Grigory Krapivinsky for help in preparation of the mRPF plasmid, V. A. Tanner Crocker for help with EM work, and Robert Malinow and Neal Waxham for comments on this manuscript.

Correspondence should be addressed to Dr. John Lisman, Brandeis University, Volen Center For Complex System, 415 South Street, Waltham, MA 02454. E-mail: lisman@brandeis.edu.

S. Carpenter's present address: University of Massachusetts Medical School, 55 Lake Avenue North, Worcester, MA 01655. E-mail: stephen.carpenter@umassmed.edu.

DOI:10.1523/JNEUROSCI.2350-04.2004

Copyright $\odot 2004$ Society for Neuroscience $\quad 0270-6474 / 04 / 249324-08 \$ 15.00 / 0$ review, see Lisman et al., 2002), it becomes critical to understand the steps after its activation. An important recent discovery is that synaptic activity causes CaMKII to translocate from the dendritic cytoplasm to the synapse both in vitro (Shen and Meyer, 1999; Shen et al., 2000) and in vivo (Gleason et al., 2003). Biochemical and EM studies indicate that activity induces an increase in CaMKII associated with postsynaptic densities (PSDs) (Strack et al., 1997; Leonard et al., 1999; Dosemeci et al., 2001). Under the conditions of these experiments, which did not have demonstrable LTP, translocation was a transient phenomenon that lasted no longer than $\sim 5$ min. A similar transience during LTP would imply that the kinase is not a memory molecule itself but rather triggers other synaptic reactions responsible for long-term changes. Several recent results, however, suggest that CaMKII translocation to synapses might be more persistent under conditions of LTP induction (Strack et al., 1997). Specifically, autophosphorylation of CaMKII on T286, a reaction that occurs during LTP, increases the affinity of CaMKII binding to synaptic molecular targets (Strack and Colbran, 1998; Gardoni et al., 1999; Strack et al., 2000a; Bayer et al., 2001; Walikonis et al., 2001; Leonard et al., 2002) and prolongs the CaMKII translocation caused by synaptic activation (Strack et al., 1997; Shen et al., 2000; Dosemeci et al., 2002). Here, we studied translocation during NMDA receptor (NMDAR)-dependent chemical LTP (cLTP) 
and found that under these conditions, CaMKII $\alpha$ translocation is persistent ( $>90 \mathrm{~min}$ ). These findings raise the possibility that the CaMKII content of synapses could act as a long-lasting memory trace of previous activity.

\section{Materials and Methods}

Slice culture. Hippocampal slice cultures were prepared and maintained similarly to the protocol described by Hayashi et al. (2000) and Otmakhov et al. (2004). Briefly, $250 \mu \mathrm{m}$ slices were prepared from postnatal day 6 (P6)-P8 Sprague Dawley rats using a Leica (Nussloch, Germany) VT 1000S vibratome. Sterile, cold $\left(4^{\circ} \mathrm{C}\right)$ modified artificial CSF (ACSF) saturated with $95 \% \mathrm{O}_{2}$ and $5 \% \mathrm{CO}_{2}$ was used during the preparation. Modified ACSF contained the following (in $\mathrm{mM}$ ): 248 sucrose, $4 \mathrm{KCl}, 1 \mathrm{CaCl}_{2}, 5$ $\mathrm{MgCl}_{2}$, and $26 \mathrm{NaHCO}_{3}$, pH 7.3, 320 mOsm. Slices were plated on the membrane of six-well plate inserts (Falcon; $0.4 \mu \mathrm{m}$ pore size) and incubated in a $\mathrm{CO}_{2}$ incubator at $36^{\circ} \mathrm{C}$ in culture medium containing MEM (cellgro; Mediatech Inc., Herndon, VA; catalog \#50-019-PB) supplemented with the following: $20 \%$ horse serum (Sigma, St. Louis, MO), $1 \mu \mathrm{g} / \mathrm{ml}$ insulin, $1 \mathrm{~mm}$ glutamine, $30 \mathrm{~mm}$ HEPES, $1 \mathrm{~mm} \mathrm{CaCl}_{2}, 2 \mathrm{~mm} \mathrm{MgSO}_{4}, 5 \mathrm{~mm}$ $\mathrm{NaHCO}_{3}, 16.5 \mathrm{~mm}$ D-glucose, and $0.5 \mathrm{~mm}$ ascorbate. The medium was changed two to three times per week.

Transfection. After 7-10 d in vitro, cultures were transfected with green fluorescent protein (GFP)-fused CaMKII $\alpha$ alone or in combination with monomeric red fluorescent protein (mRFP). GFP-CaMKII $\alpha$ (wild type)-containing vectors were generous gifts from Dr. Tobias Meyer (Shen and Meyer, 1998, 1999) and Dr. Neal Waxham (Kim et al., 2004). Biochemical properties of GFP-CaMKII $\alpha$ were shown previously to be similar to those of the native kinase (Shen and Meyer, 1998; Kim et al., 2004). The coding sequence of mRFP (a generous gift from Dr. Roger Tsien) (Campbell et al., 2002) was inserted in the enhanced GFP (EGFP)-C1 plasmid (Clontech, Cambridge, UK) in place of EGFP. Gene gun transfection was performed according to the instructions of the manufacturer (Bio-Rad, Hercules, CA), except $100 \mu \mathrm{m}$ nylon mesh was inserted as a pressure-deflecting screen (Lo et al., 1994). Slices were used for electrophysiological and imaging experiments at days 1-6 (typically days 2-3) after the transfection. (Experiments started when expressed protein produced the minimal fluorescence sufficient for imaging.)

Electrophysiology. For an experiment, a single cultured slice was cut out from the cell culture insert and placed in the recording chamber mounted on the microscope stage. Slices were continuously superfused $(2 \mathrm{ml} / \mathrm{min}$ ) with ACSF containing the following (in mM): $124 \mathrm{NaCl}, 2.5 \mathrm{KCl}, 4 \mathrm{CaCl}_{2}, 4 \mathrm{MgCl}_{2}, 1.25$ $\mathrm{NaH}_{2} \mathrm{PO}_{4}, 26 \mathrm{NaHCO}_{3}$, and 0.0062 -Cl-adenosine, balanced with $95 \% \mathrm{O}_{2}$ and $5 \% \mathrm{CO}_{2}, \mathrm{pH}$ 7.4. For extracellular stimulation and field potential recording, glass pipettes $(300 \mathrm{k} \Omega$ ) filled with ACSF were positioned in the stratum radiatum of the CA1 region. Single-shock stimuli (1-5 $\mu \mathrm{A}, 100 \mu \mathrm{sec}$ duration square pulse) were delivered every $5 \mathrm{~min}$ through current isolation units. Recorded signals were amplified 20,000 times, filtered (0.5-1000 $\mathrm{Hz}$ ), digitized $(5-10 \mathrm{kHz})$, and analyzed using custom software written in Axobasic. Recordings were performed at room temperature $\left(22-24^{\circ} \mathrm{C}\right)$.

Chemical LTP. LTP was induced by bath application of the adenylyl cyclase activator forskolin $(50 \mu \mathrm{M})$ and the phosphodiesterase inhibitor rolipram $(0.1 \mu \mathrm{M})$ in ASCF that contained neither $\mathrm{Mg}^{2+}$ nor 2-Cl-adenosine (0 Mg ACSF). This solution was applied for $16 \mathrm{~min}$, and the electrical stimulation was stopped for this period and for 30 min afterward. An increase in field EPSP (fEPSP) slope of $>20 \%$ of the baseline for at least $45 \mathrm{~min}$ after resumption of synaptic stimulation was considered to be cLTP. During the period of drug application, we observed spontaneous, periodic (every 5-30 sec), giant field potentials (up to 10-fold the amplitude of normal fEPSPs; data not shown), indicating the occurrence of synchronous spontaneous activation of cells. In a similar protocol for cLTP (Makhinson et al., 1999), spontaneous bursting of CA3 neurons was demonstrated to be a requirement for the CLTP induction. Because of this dependence on spontaneous presynaptic bursting and because slice cultures have extensive CA3-CA1 connections (because of regeneration) this method induces cLTP in a large fraction of synapses (Otmakhov et al., 2004).

Imaging. Single fluorophore imaging was performed using a Noran confocal unit built on an Olympus Optical (Tokyo, Japan) upright BX50WI microscope and 60× LUMPlanFL [0.9 numerical aperture (NA), water immersion (WI)] objective, or a conventional fluorescence microscope equipped with a $100 \times$ LUMPlanFL (1.0 NA, WI) objective and MetaMorph software (Universal Imaging, Downingtown, PA). Imaging experiments with double protein expression were performed on a Leica upright confocal microscope equipped with a $40 \times(0.8 \mathrm{NA}, \mathrm{WI})$ objective and an $\mathrm{Ar}-\mathrm{Kr}$ laser. Green and red images were taken consecutively at each focal plan using 488 and 543 excitation wavelengths and 500-550 and 590-650 emission, respectively. Cross-channel fluorescence bleeding was negligible and therefore not corrected. In all of the cases, the images were obtained from dendritic branches close to the slice surface (5-30 $\mu \mathrm{m})$ and under exactly the same conditions as electrophysiological experiments. Normally, stacks of 15-20 images with $0.5 \mu \mathrm{m}$ focal steps were collected. On the confocal setups, an additional $1.8-4 \times$ digital zoom was used, rendering $0.064-0.146 \mu \mathrm{m} /$ pixel resolution in all of the setups.

Image analysis. Experiments with single protein expression (GFP-CaMKII $\alpha$ ) were analyzed using MetaMorph software. Each image was low-pass-filtered, and projection images were constructed for each time point (see Fig. 3A). Each spine, identified as dendritic protrusion of up to $1.5 \mu \mathrm{m}$ in length, was given a number. Images displayed with pseudocolor representation of intensity were visually compared, and spines increasing fluorescence after the drug application were identified (see Fig. $3 B, C$ ). During subsequent quantitative analysis, maximal spine fluorescence, $F$, for each time point was calculated (see Fig. $3 D$ ), corrected for photobleaching, and normalized relative to the average $F$ before drug application, and $\Delta F / F$ was plotted against the time (see Fig. $3 E$ ). The rate of photobleaching was calculated from similar spine analysis in control experiments (without drug application). All of the analyzed spines were divided into three groups as follows: the first group (transient and persistent translocation) comprised all spines in which fluorescence increased at least $8 \%$ above the baseline during drug application and remained at this level for 10 min or more after the drug washout; the second group (persistent translocation) was a subset of group 1 in which the $\Delta F / F$ increase persisted for at least $60 \mathrm{~min}$; and the third group (no translocation) consisted of spines not falling into group 1 or 2 .

Experiments with double protein expression of GFPCaMKII $\alpha$ and mRFP were analyzed using custom software (IGOR Pro; Wavemetrics, Lake Oswego, OR). To eliminate background and out-of-focus fluorescence, a binary mask was constructed for each image in a given stack of $z$-plane images (see Fig. 1 ). The mask covered all of the pixels below an arbitrarily chosen threshold equal to double the background fluorescence. Regions of interest (ROIs) were drawn around spines and nearby den- 
drites. The average fluorescence of pixels within this region but not covered by the mask was measured for each image in the $z$-stack (see Fig. 1C). The maximal average fluorescence in the $z$-stack was corrected for background fluorescence and then used for additional analysis of spine (s) or dendritic (d) regions. The spine/dendrite (s/d) fluorescence ratio was calculated for each spine for both green and red channels, normalized relative to its value before induction of CLTP and plotted over time (see Fig. $5 C-E$ ). All of the data are presented as mean \pm SE. Statistical significance of differences was calculated using Student's paired $t$ test, nonparametric ANOVA, or the Kolmogorov-Smirnov test.

Estimation of the bound GFP-CaMKII $\alpha$ pool in spines. mRFP should be in equilibrium between spine and dendrite because the spine/dendrite fluorescent ratio of free monomeric fluorescent proteins is no different from that of small fluorescent dyes (Piccini and Malinow, 2002). These dyes have been shown to equilibrate between spine and dendrite within milliseconds (Svoboda et al., 1996). Cellular GFP-CaMKII $\alpha$ may exist in both a soluble, freely diffusible state and in a bound state. In the dendrite, it is likely to be mostly free because it is homogeneously distributed and because its recovery after photobleaching occurs within seconds (Shen and Meyer, 1999; Otmakhov et al., 2001). Furthermore, the major known binding proteins for GFP-CaMKII $\alpha$ (NMDA receptors, densin-180/ $\alpha$-actinin) are concentrated at spines. It follows that if all of the GFP-CaMKII $\alpha$ in the spine is free, the $\mathrm{s} / \mathrm{d}$ ratio for green, $(\mathrm{s} / \mathrm{d})_{\mathrm{G}}$, should be equal to that for red, $(\mathrm{s} / \mathrm{d})_{\mathrm{R}}$. A larger $(\mathrm{s} / \mathrm{d})_{\mathrm{G}}$ than $(\mathrm{s} / \mathrm{d})_{\mathrm{R}}$ would indicate the presence of a bound spine pool. In this case, the amount of spine fluorescence produced by the soluble GFP-CaMKII $\alpha$ pool can be estimated as $\mathrm{d}_{\mathrm{G}} \cdot(\mathrm{s} / \mathrm{d})_{\mathrm{R}}$, and the fluorescence from the bound pool can be estimated as the difference between the total spine signal and the signal from the soluble pool, $\mathrm{s}_{\mathrm{G}}-\mathrm{d}_{\mathrm{G}} \cdot(\mathrm{s} / \mathrm{d})_{\mathrm{R}}$. The fraction of GFP-CaMKII $\alpha$ bound in the spine is thus $\left[\mathrm{s}_{\mathrm{G}}-\mathrm{d}_{\mathrm{G}} \cdot(\mathrm{s} / \mathrm{d})_{\mathrm{R}}\right] / \mathrm{s}_{\mathrm{G}}$.

EM immunolabeling. Hippocampal slice cultures were fixed in $4 \%$ paraformaldehyde and $4 \%$ sucrose in PBS overnight at $4^{\circ} \mathrm{C}$ and then stored in $20 \times$ dilution of the same fixative. The CA1 region was dissected into thin strips, with the orientation carefully monitored so that the stratum radiatum can be readily identified thereafter. Samples were blocked and permeabilized with $5 \%$ normal goat serum and $0.1 \%$ saponin in PBS for $1 \mathrm{hr}$, incubated in primary antibody (monoclonal antibody against CaMKII $\alpha$; clone 6G9-2; Chemicon, Temecula, CA) for $1 \mathrm{hr}$, washed, and incubated in secondary antibody conjugated to 1.4 nm gold particles (nanogold; Nanoprobes, Yaphank, NY) for 1 $\mathrm{hr}$, washed, and fixed in 2\% glutaraldehyde in PBS, washed in water, and silver-enhanced (HQ silver enhancement kit; Nanoprobes). Samples were then treated with $0.2 \% \mathrm{OsO}_{4}$ and $0.25 \%$ uranyl acetate, dehydrated through a graded series of ethanol, and embedded in epoxy resins.

Morphometry of PSD-associated CaMKII $\alpha$ labeling. Samples were processed after a blind coding, and synapses were sampled from stratum radiatum. An image of every encountered synapse was captured with a CCD digital camera system (XR-100; Advanced Microscopic Techniques, Danvers, MA). Prints at $150,000 \times$ final enlargement were coded, and measurements were performed blind in random order using NIH Image 1.61. Data were analyzed by nonparametric ANOVA. The PSD area was

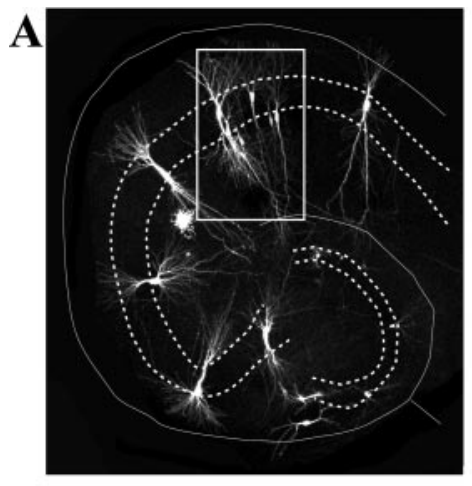

C

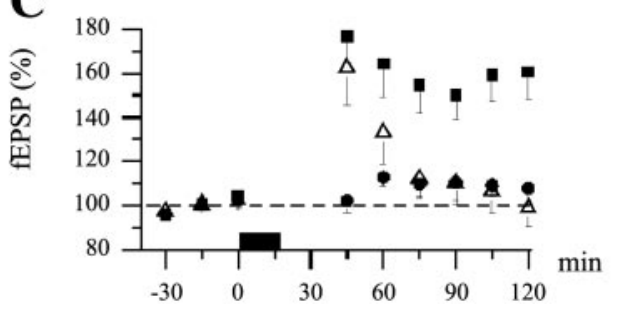

Figure 2. Induction of chemically induced, NMDA receptor-dependent LTP in slices transfected with GFP-CaMKII $\alpha$. A, GFP-CaMKII $\alpha$ expression in hippocampal neurons in slice culture $13 \mathrm{~d}$ in vitro at day 4 after transfection. $B$, Zoomed image of the region indicated in $A$ shows expression in all of the cellular compartments. Scale bar, $40 \mu \mathrm{m}$. C, Summary graphs of synaptic potentiation in control ACSF without APV $(\square)$, in the presence APV, $100 \mu \mathrm{M}(\triangle)$, or when no induction procedure was applied ( $)$. The induction procedure is indicated by the horizontal bar. Each data point is an average of three responses. D, Average of five fEPSPs before (thin line) and 120 min after (thick line) induction. Calibration: $50 \mu \mathrm{V}, 20 \mathrm{msec}$.

marked by tracing its cytoplasmic outline on the prints, including the associated dense material, and enclosing the area with the postsynaptic membrane (Dosemeci et al., 2002). To calculate the amount of gold label over a unit length of the postsynaptic membrane, all of the particles within or contacting the outline of each PSD were counted, and this number was divided by the length of the corresponding postsynaptic membrane. To avoid effects of variation in labeling intensity from different runs, only samples from the same immunocytochemistry run were compared against each other.

Drugs. Forskolin (Alomone Labs, Jerusalem, Israel) and Rolipram (Sigma) were dissolved in DMSO at concentrations of 50 and $1 \mathrm{~mm}$, respectively, and stored at $-70^{\circ} \mathrm{C}$ before use. The final concentration of DMSO in ACSF was $0.11 \%$. DL-2-Amino-5phosphono-valeric acid (APV) was dissolved in water (20 mM) 


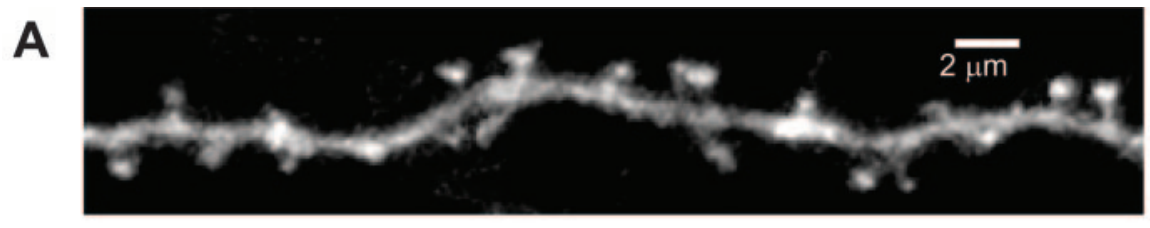

B

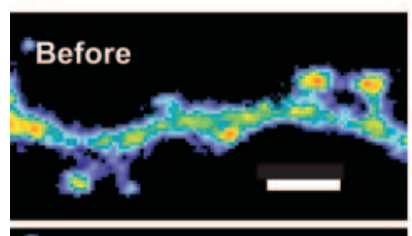

$\mathrm{C}_{\text {Before }}$
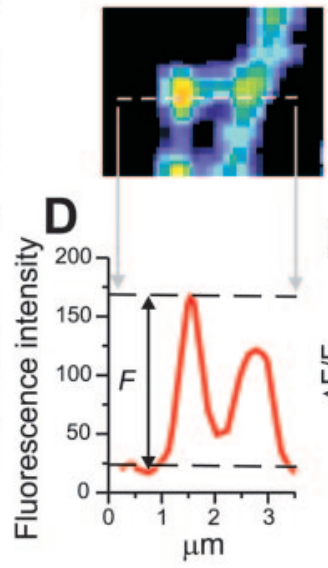

$\mathbf{F}$

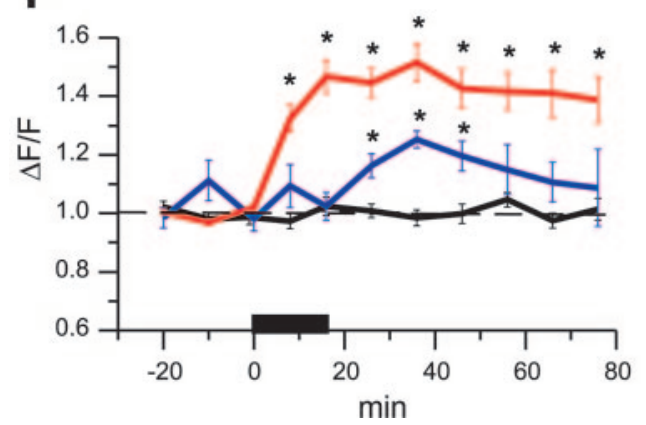

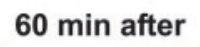

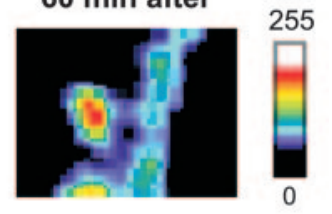

E

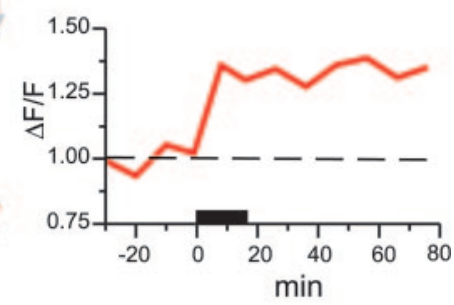

G

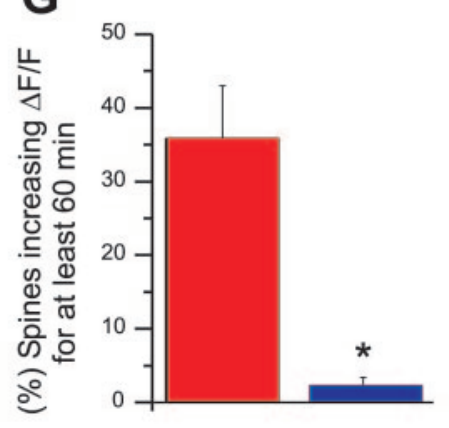

Figure 3. Persistent NMDA receptor-dependent accumulation of GFP-CaMKII $\alpha$ in spines during CLTP. A, Projection image of a dendritic branch of a CA1 pyramidal neuron showing clearly identifiable spines. $B$, Images of a smaller dendritic region taken before and $60 \mathrm{~min}$ after application of CLTP-inducing protocol. Fluorescence intensity is represented in pseudocolor. Arrows indicate spines with an increase in $\Delta F / F$. Scale bars: $A, B, 2 \mu \mathrm{m}$. $C$, Images of a single spine before and after the induction procedure. Inset, Pseudocolor lookup table. $D$, Graph of $F$ along the line indicated in $C$. $E$, Change of the fluorescence intensity $(\Delta F / F)$ over time for spine in $C$. Horizontal bar here and in $F$ indicates drug application. $F$, Summary graph showing average temporal dynamics of fluorescence for all 135 of the spines with increasing $\Delta F / F$ in 11 experiments in which the LTP induction protocol was applied without APV (red) or in the presence of APV ( 7 spines in 5 experiments, blue). Black indicates summary graph for all 43 of the spines in three experiments in which no drugs were applied. G, Percentage of spines increasing $\Delta F / F$ for at least 60 min after drug application in the presence or absence of APV; asterisks indicate statistically significant data.

and stored at $4^{\circ} \mathrm{C}$ before being used at a final concentration of 100 $\mu \mathrm{M}$. 2-Cl-adenosine was dissolved in water $(3 \mathrm{~mm})$ and stored at $4^{\circ} \mathrm{C}$ before being used at final concentration of $6 \mu \mathrm{M}$.

\section{Results}

\section{Induction of cLTP}

We used a chemical method for the induction of LTP at a large fraction of synapses, making this preparation ideal for simultaneously monitoring translocation at many synapses (Otmakhov et al., 2004). The method is similar to that used in acute slices (Makhinson et al., 1999) but modified for slice cultures (see Materials and Methods). This cLTP requires presynaptic activity, is NMDAR-dependent, and occludes with tetanically induced LTP (Makhinson et al., 1999; Otmakhov et al., 2004). In our experiments, cLTP, measured as a persistent increase in the slope of the fEPSPs, was reliably induced in 11 of 12 experiments and was substantial in size $(170 \pm 11 \%$ of the basal level at $120 \mathrm{~min}$ after induction; $p<0.01$ ) (Fig. $2 C$ ). In the presence of an NMDAR antagonist, potentiation was only transient; by $60 \mathrm{~min}$, the response was no different from the baseline $(110 \pm 7 \% ; n=7 ; p>$ 0.05) (Fig. 2C). In control experiments in which the induction protocol was not applied, there was no significant change in the response $(98 \pm 9 \%$ at $120 \mathrm{~min} ; n=8 ; p>$ 0.05) (Fig. 1C).

\section{cLTP induction results in persistent} accumulation of CaMKII in spines To visualize CaMKII movements during cLTP, we expressed wild-type CaMKII $\alpha$ fused with GFP in cultured hippocampal slices (see Materials and Methods). Neurons expressing GFP-CaMKII $\alpha$ were healthy and had abundant dendritic spines (Figs. 2A,B, $3 A$ ). We measured GFP-CaMKII $\alpha$ accumulation in spines because these are sites of individual excitatory synapses. Spine fluorescence $(F)$ was calculated as the difference between the maximum fluorescence in a spine and the background fluorescence (Fig. $2 C, D)$. After induction of cLTP, fluorescence in many spines significantly increased (Fig. $3 B$ ). An example of clear and persistent GF$\mathrm{P}-\mathrm{CaMKII} \alpha$ accumulation in a spine head is shown in Figure 3, $C$ and E. On average, in each experiment, $41.1 \pm 6.6 \%$ of spines ( 135 of 331 imaged spines in 11 experiments) showed an increase in $\Delta F / F$ of at least $8 \%$ (up to $500 \%$ ) after the cLTP-inducing procedure. Although in some spines the increase was transient, in a large fraction of spines $(36.5 \pm 7.1 \% ; 126$ of 331$)$, the increase was long-lasting (at least $60 \mathrm{~min}$ ) (Fig. $3 G$ ). The summary graph for all 135 of the spines increasing $\Delta F / F$ shows that the increase was $1.40 \pm 0.08$-fold from the baseline at $60 \mathrm{~min}$ after induction (Fig. 3 F). This is significantly different from the average increase for all of the spines in experiments with no drug application (43 spines in three experiments; $1.01 \pm 0.08 ; p<0.01$ ) (Fig. 3F).

We also analyzed the spines that did not show an increase in $\Delta F / F$ of GFP-CaMKII $\alpha$. The average $\Delta F / F$ in those spines showed a small but statistically significant decrease when compared with control experiments $(0.92 \pm 0.03$ vs $0.99 \pm$ 0.03 in controls at $60 \mathrm{~min}$ after the induction; $n=112 ; p<0.05)$. This decrease still occurred in the presence of APV $(0.86 \pm 0.03$ at 60 min after the induction; $n=51 ; p<0.05)$, suggesting that there is an activity-dependent process that can reduce spine CaMKII content through a non-NMDA mechanism.

\section{CaMKII accumulation is NMDAR-dependent}

Because cLTP measured electrophysiologically was NMDARdependent, we tested whether the persistent accumulation of GFP-CaMKII $\alpha$ in spines also depends on NMDAR activation and found that it does. In the presence of $100 \mu \mathrm{M}$ APV, an increase in $\Delta F / F$ occurred only in 7 of 89 spines and typically was 
only transient, returning to baseline within $40 \mathrm{~min}$ after induction (Fig. $3 F)(1.25 \pm 0.03$ vs $0.99 \pm 0.03 ; p<0.01$ at $20 \mathrm{~min}$; and $1.14 \pm 0.09$ vs $1.05 \pm 0.02 ; p>0.05$ at $40 \mathrm{~min})$. Only in 2 of 89 imaged spines $(2.3 \pm 1.1 \%$; five experiments) was the increase long-lasting (60 min), and this was significantly smaller from experiments with cLTP induction $(p<0.01)$ (Fig. $3 G)$. Therefore, like cLTP induction (Fig. 2C), the persistent translocation of GFP-CaMKII $\alpha$ into spines is NMDAR-dependent.

\section{Both spine volume and CaMKII bound fraction increases} after cLTP induction

The CaMKII in spines consists of multiple pools: some CaMKII is soluble, whereas other pools are bound to molecular targets in spines (Strack et al., 1997; Strack and Colbran, 1998; Shen and Meyer, 1999; Otmakhov et al., 2001; Walikonis et al., 2001; Hudmon and Schulman, 2002). Because spine size may increase after LTP induction (Luscher et al., 2000; Piccini et al., 2003; Matsuzaki et al., 2004), there is the possibility that the increase in spine fluorescence (Fig. 3) is attributable to an increase in the soluble GFP-CaMKII $\alpha$ pool that is secondary to a volume change. Alternatively, part or all of the increase may be a result of an increase in a bound pool. To distinguish between these possibilities, we coexpressed GFP-CaMKII $\alpha$ together with mRFP, which is a freely diffusible soluble protein and can serve as a marker of cell volume (Piccini and Malinow, 2002) (see Materials and Methods). The average red and green fluorescence was measured in spines and nearby dendritic regions (Fig. 1). First, we asked whether under basal conditions the GFP-CaMKII $\alpha$ fluorescence in spines could be fully accounted for by the free pool; in this case, the s/d fluorescence ratio for GFPCaMKII $\alpha$ should be the same as that for mRFP (Piccini and Malinow, 2002). We found that even under basal conditions the average s/d ratio for GFP-CaMKII $\alpha$ was significantly higher than that for mRFP $(0.87 \pm 0.06$ vs $0.43 \pm 0.03 ; p<0.01 ; n=$ 20) (Fig. 4) indicating the existence of a bound pool in spines. We then asked whether the increase in spine GFPCaMKII $\alpha$ fluorescence during cLTP could be accounted for by an increase in spine volume. Our finding was that spines that showed an increase in GFP-CaMKII $\alpha$ $\mathrm{s} / \mathrm{d}$ ratio also showed a significant rise in $\mathrm{mRFP} s / \mathrm{d}$ ratio $(1.18 \pm 0.06$-fold at $50-90$ min after induction; $p<0.01 ; 18$ spines; two experiments), indicating an enlargement of spine volume during cLTP (Fig. $5 D)$. This is in agreement with reports (Luscher et al., 2000; Matsuzaki et al., 2003; Piccini et al., 2003) indicating that spine volume may increase after LTP. On average, however, the increase in $\mathrm{mRFP}$ s/d ratio was considerably smaller than that for GFP-CaMKII $\alpha$ (1.69 \pm 0.1 -fold; $p<0.01)$. In control experiments without cLTP induction, the s/d ratio was stable for both green and red channels: at $46-66 \mathrm{~min}$, this ratio was $1.07 \pm 0.04$-fold of the initial baseline for GFP-CaMKII $\alpha$ and $1.06 \pm 0.04$ for mRFP (29 spines; $p>0.05$ for both) (Fig. $5 E$ ). We concluded that the increase in spine GFP-CaMKII $\alpha$ fluorescence cannot be fully ac-
A

B
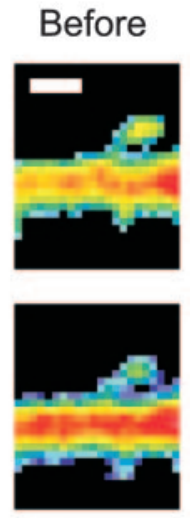

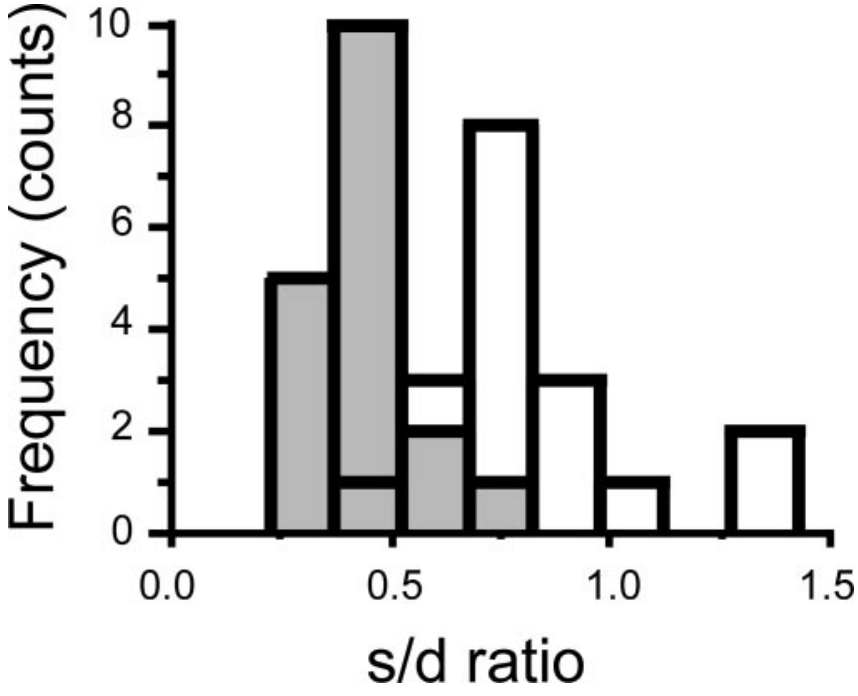

Figure 4. In many spines, GFP-CaMKII $\alpha$ is already accumulated under basal conditions. Distribution of $\mathrm{s} / \mathrm{d}$ fluorescence intensity for red (gray bars) and green (open bars) channels in control conditions $(n=20)$ is shown. The average $s / d$ fluorescence ratio for the green channel was significantly larger, $0.87 \pm 0.06$, than that for the red channel, $0.43 \pm 0.03(p<0.01)$, indicating that bound GFP-CaMKII $\alpha$ is already present in spines before the induction of $\mathrm{CLTP}$. The calculated fraction of the GFP-CaMKII $\alpha$ bound fraction in spines is 0.50 or $50 \%$ (see Materials and Methods).
LTP $70 \mathrm{~min}$
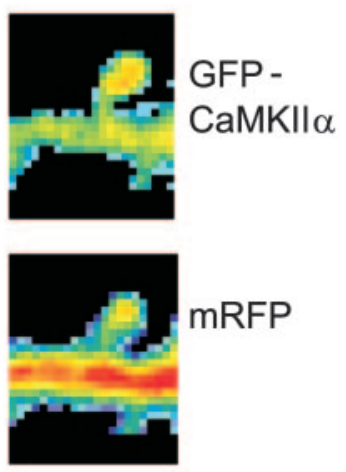
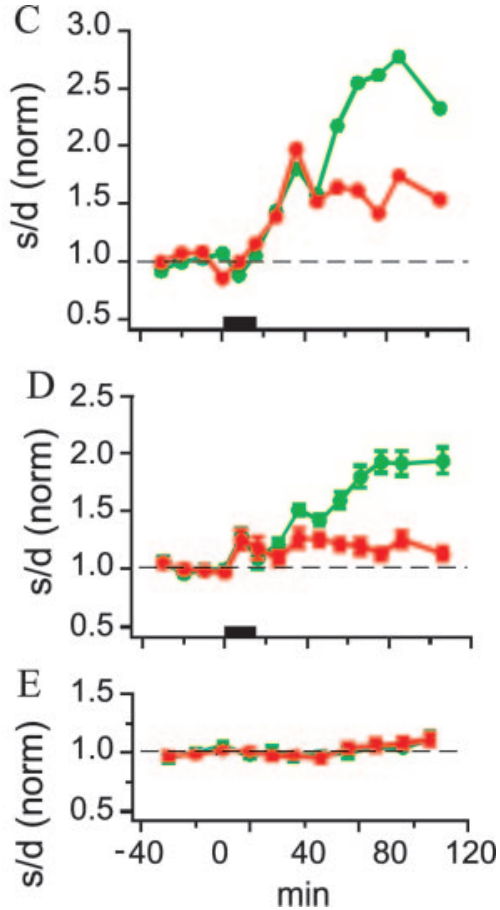

Figure 5. Increase in spine CaMKII accumulation cannot be accounted for by an increase in spine volume. $A$, Expression of GFP-CaMKII $\alpha$ (green) and mRFP (red) in the same dendritic branch. B, GFP-CaMKIl $\alpha$ (top) and mRFP (bottom) expression in a single spine before and $70 \mathrm{~min}$ after LTP induction, shown in pseudocolor. $C$, Change over time in s/d fluorescence ratio for GFP-CaMKII $\alpha$ (green) and mRFP (red) for the spine shown in B. D, Summary graph for 18 spines showing an increased s/d ratio. The horizontal bar indicates CLTP induction. norm, Normalized. E, Summary graph from three control experiments without CLTP induction (29 spines). Scale bars: $A, 2 \mu \mathrm{m} ; B, 1 \mu \mathrm{m}$. counted for by an increase in spine volume and is likely to reflect both an increase in spine volume and an increase in the bound pool fraction within the spine.

We estimated the fraction of GFP-CaMKII $\alpha$ that is bound (see Materials and Methods). Induction of CLTP produces a large 

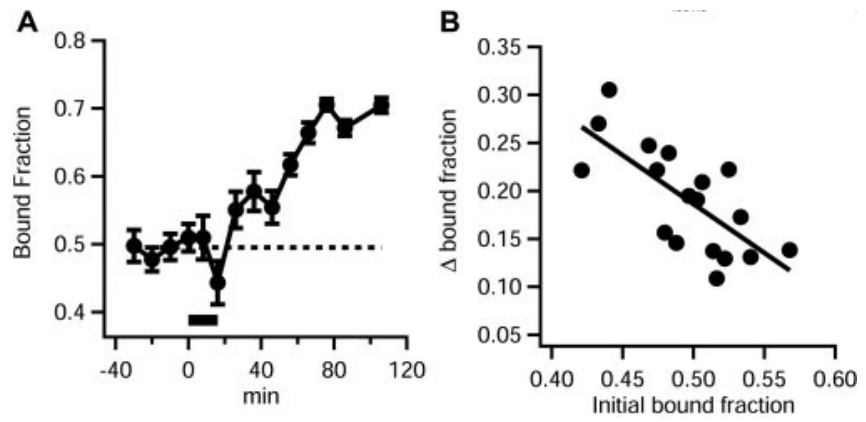

Figure 6. The fraction of the GFP-CaMKII $\alpha$ bound pool in spines increases after induction of LTP. A, Dynamic of change in the fraction of bound CaMKIl $\alpha$ pool in spines. The bound fraction was calculated as described in Materials and Methods. The horizontal bar indicates the period of CLTP induction. $B$, The CLTP-induced change in the bound fraction of CaMKII is reversely correlated with the initial value before CLTP induction. Each filled circle represents the result of a single experiment.

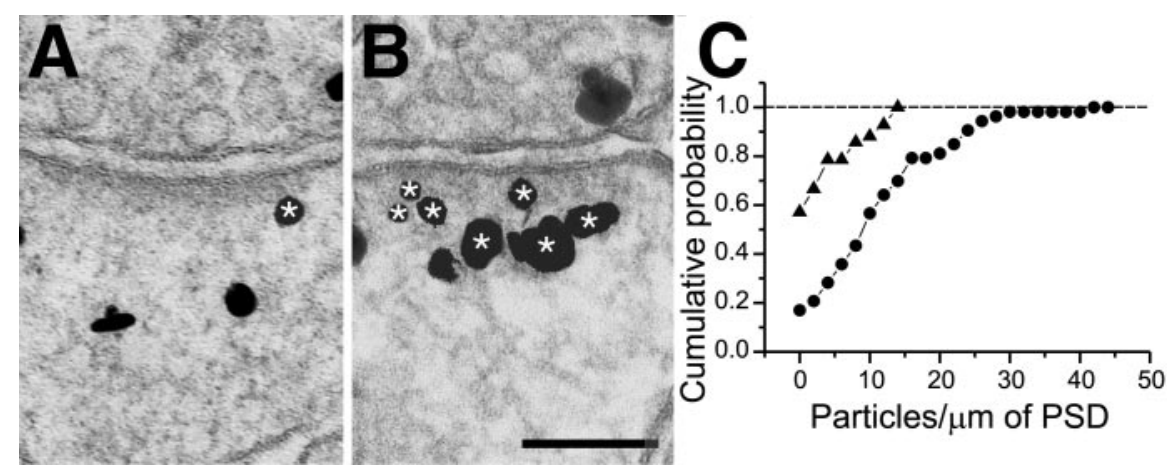

Figure 7. $\quad \mathrm{CLTP}$ induction results in persistent accumulation of CaMKIl $\alpha$ at the PSD. $A, B$, Electron micrographs of hippocampal synapses labeled for CaMKII $\alpha$ under control conditions $(A)$ and $1 \mathrm{hr}$ after induction of $\mathrm{CLTP}(B)$. Silver-enhanced gold particles appear as irregular black grains. Asterisks indicate grains counted as PSD-associated CaMKII labeling. Scale bar, $100 \mathrm{~nm}$. C, Cumulative distribution of densities of gold label for CaMKII $\alpha$ at individual PSD from slice cultures under control conditions ( $(\boldsymbol{\Delta})$ and after induction of chemical LTP $(\mathbf{O})$.

and persistent (at least $90 \mathrm{~min}$ ) increase of the bound fraction from $0.5 \pm 0.01$ at the basal level to $0.69 \pm 0.01$ during cLTP (at 50-90 min; $n=18 ; p<0.01$ ) (Fig. 6A). Interestingly, the amount of change in the bound fraction of GFP-CaMKII $\alpha$ driven by cLTP induction shows a strong reverse correlation $(r=-0.72)$ with the initial value of the fraction before cLTP (i.e., the larger the initial bound pool, the smaller the increase produced by cLTP) (Fig. 6B). This is reminiscent of a similar reverse correlation between the initial value of quantal size and the change in quantal size during LTP (Liao et al., 1992).

\section{Persistent increase in CaMKII density at the PSD}

The pool of CaMKII within the PSD is of particular importance because of its ability to modulate AMPA channels (Barria et al., 1997; Strack et al., 1997; Derkach et al., 1999; Leonard et al., 1999) and other regulatory proteins (Kennedy, 1998; Lisman et al., 2002). We used EM immunogold methods to determine whether LTP induction causes a persistent change in the levels of CaMKII $\alpha$ in this pool. Immediately after cLTP induction, there was a significant increase in CaMKII $\alpha$ labeling associated with the PSD compared with control ( $>40$ synapses from two slices in each condition). This change persisted for at least $1 \mathrm{hr}$ after cLTP induction and was approximately threefold larger than in control slices $(12.3 \pm 1.3$ grains $/ \mu \mathrm{m} ; n=53$; vs $3.4 \pm 0.7$ grains $/ \mu \mathrm{m} ; n=$ $42 ; p<0.001$; quantification performed blind), indicating that cLTP increases the association of CaMKII $\alpha$ with the dense material of the PSD (Fig. $7 \mathrm{~B}, C$ ). In contrast, the CaMKII $\alpha$ labeling in parallel con- trol slices was primarily dispersed in the cytoplasm of the spine (Fig. $7 A$ ). In particular, although after cLTP, 34\% of PSDs had 15 or more grains per micrometer, not a single control PSD had this amount of labeling. Consistent with our light microscopy results, we found that the increase in PSD-associated CaMKII $\alpha$ labeling $1 \mathrm{hr}$ after cLTP induction was blocked by APV: without APV there was more than twice the PSD-associated CaMKII $\alpha$ labeling than in slices treated with $100 \mu \mathrm{M}$ APV $(4.1 \pm 0.8$ grains $/ \mu \mathrm{m} ; n=36$; vs $1.8 \pm 0.4$ grains/ $\mu \mathrm{m} ; n=53$; $p<0.05$; two slices in each group).

\section{Discussion}

Our results indicate that cLTP induction produces a translocation of CaMKII $\alpha$ to synaptic spines that is persistent ( $>90 \mathrm{~min}$ ). Furthermore, observations at the EM level demonstrate that part of the accumulation is attributable to a persistent increase of CaMKII $\alpha$ levels in the PSD. When NMDA channels are blocked, persistent translocation observed both at the light and EM levels is blocked. There thus appear to be two forms of translocation, a transient form (Shen and Meyer, 1999; Shen et al., 2000; Dosemeci et al., 2001; Gleason et al., 2003) that may occur under some experimental conditions and a persistent form that occurs under conditions that induce LTP.

The available evidence suggests a mechanism that determines whether translocation is persistent or transient. It is known that blocking NMDA channels reduces activity-dependent $\mathrm{Ca}^{2+}$ elevation (Yuste et al., 2000) and results in a lowered level of CaMKII autophosphorylation on T286 (Fukunaga et al., 1993; Barria et al., 1997). Previous work also shows that translocation and binding of CaMKII to the PSD proteins (NMDA channel, densin-180/ $\alpha$ actinin) can occur without autophosphorylation on T286 (Strack et al., 1997, 2000b; Shen et al., 1998; Leonard et al., 1999; Shen and Meyer, 1999; Bayer et al., 2001; Walikonis et al., 2001) but that autophosphorylation increases the binding affinity (Strack and Colbran, 1998; Gardoni et al., 1999; Leonard et al., 1999, 2002; Strack et al., 2000a; Bayer et al., 2001; Walikonis et al., 2001). Consistent with this interpretation, the transient form of translocation can be greatly prolonged by a mutation (T286D) that imitates this autophosphorylation or by enhancing and maintaining autophosphorylation with phosphatase inhibitors (Shen et al., 2000; Dosemeci et al., 2002). It thus seems quite possible that enhanced phosphorylation of CaMKII during conditions that lead to LTP induction may be what converts the translocation from a transient to a persistent form. Consistent with this hypothesis, the levels of CaMKII normally found in the PSD are greatly reduced by a mutation (T306D) (Elgersma et al., 2002) that inhibits kinase activation and autophosphorylation on T286 (Hudmon and Schulman, 2002). Together, these results raise the possibility that the high levels of CaMKII normally found in the PSD are the result of persistent translocation triggered by LTP-like events that occur during the animal's experience.

\section{Change in spine size and structure}

A fundamental question is whether synaptic strengthening involves an enlargement of existing synapse-spine structures or a 
modification of these structures. Our results point to both types of changes. The finding of a persistent increase in spine size after LTP (Fig. 5) adds to the accumulating evidence that LTP produces spine enlargement (Luscher et al., 2000; Matsuzaki et al., 2003; Piccini et al., 2003). However, if the CaMKII addition to the PSD were only a result of enlarging the PSD area, leaving the existing PSD unaltered, the density of CaMKII would not increase. Our results show, however, that the density of CaMKII in the PSD increases after LTP. We conclude that there is at least one type of CaMKII change that leads to the modification of existing structures.

The increase we observe in the bound pool of CaMKII in spines may not only be because of binding to the PSD. In addition to binding to the PSD proteins, CaMKII $\alpha$ may also indirectly (through CaMKII $\beta$ and $\alpha$-actinin) bind to F-actin at spine heads, the amount of which increases during LTP (Luscher et al., 2000; Fukazawa et al., 2003). Other recently discovered (Dhavan et al., 2002; Illario et al., 2003; Krapivinsky et al., 2004) and yet unknown binding partners might also contribute to CaMKII spine accumulation. Therefore, LTP may involve changes in multiple spine pools.

\section{CaMKII as a memory trace}

Our results show that synaptic CaMKII $\alpha$ is increased for $>1 \mathrm{hr}$ after cLTP induction, a change that could function as a memory trace. It will now be of importance to determine the duration of this change and its functional role. CaMKII can phosphorylate many PSD substrates (Fink and Meyer, 2002; Lisman et al., 2002), most notably GluR1 (Barria et al., 1997), phosphorylation of which may contribute to synaptic strengthening by enhancing the single-channel conductance (Derkach et al., 1999). However, eliminating CaMKII-dependent phosphorylation of GluR1 (Lee et al., 2003) or blocking CaMKII activity after induction (Malinow et al., 1989; Otmakhov et al., 1997) does not strongly affect LTP maintenance, suggesting that ongoing phosphorylation of these substrates is not necessary for the maintenance of LTP. Other actions of CaMKII may be structural; once bound in the PSD, the kinase may act as a seed for the binding of other proteins that strengthen the synapse. These proteins could serve as slots for the binding of additional AMPA channels (Lisman and Zhabotinsky, 2001; Shi et al., 2001), the density of which, like CaMKII, varies greatly from synapse to synapse (Nusser et al., 1998). The demonstration of persistent accumulation of CaMKII at synapses now points to a critical next step in the elucidation of synaptic memory: if this accumulation is indeed a synaptic memory trace, agents that interfere with the mechanisms of this accumulation should reverse the translocation and thereby lead to a reversal of LTP.

\section{References}

Barria A, Muller D, Derkach V, Griffith LC, Soderling TR (1997) Regulatory phosphorylation of AMPA-type glutamate receptors by CaM-KII during long-term potentiation. Science 276:2042-2045.

Bayer KU, De Koninck P, Leonard AS, Hell JW, Schulman H (2001) Interaction with the NMDA receptor locks CaMKII in an active conformation. Nature 411:801-805.

Bliss TV, Collingridge GL (1993) A synaptic model of memory: long-term potentiation in the hippocampus. Nature 361:31-39.

Campbell RE, Tour O, Palmer AE, Steinbach PA, Baird GS, Zacharias DA, Tsien RY (2002) A monomeric red fluorescent protein. Proc Natl Acad Sci USA 99:7877-7882.

Derkach V, Barria A, Soderling TR (1999) $\mathrm{Ca}^{2+} /$ calmodulin-kinase II enhances channel conductance of alpha-amino-3-hydroxy-5-methyl-4isoxazolepropionate type glutamate receptors. Proc Natl Acad Sci USA 96:3269-3274.
Dhavan R, Greer PL, Morabito MA, Orlando LR, Tsai LH (2002) The cyclin-dependent kinase 5 activators p35 and p39 interact with the $\alpha$-subunit of $\mathrm{Ca}^{2+} /$ calmodulin-dependent protein kinase II and $\alpha$-actinin-1 in a calcium-dependent manner. J Neurosci 22:7879-7891.

Dosemeci A, Tao-Cheng JH, Vinade L, Winters CA, Pozzo-Miller L, Reese TS (2001) Glutamate-induced transient modification of the postsynaptic density. Proc Natl Acad Sci USA 98:10428-10432.

Dosemeci A, Vinade L, Winters CA, Reese TS, Tao-Cheng JH (2002) Inhibition of phosphatase activity prolongs NMDA-induced modification of the postsynaptic density. J Neurocytol 31:605-612.

Elgersma Y, Fedorov NB, Ikonen S, Choi ES, Elgersma M, Carvalho OM, Giese KP, Silva AJ (2002) Inhibitory autophosphorylation of CaMKII controls PSD association, plasticity, and learning. Neuron 36:493-505.

Fink CC, Meyer T (2002) Molecular mechanisms of CaMKII activation in neuronal plasticity. Curr Opin Neurobiol 12:293-299.

Fukazawa Y, Saitoh Y, Ozawa F, Ohta Y, Mizuno K, Inokuchi K (2003) Hippocampal LTP is accompanied by enhanced F-actin content within the dendritic spine that is essential for late LTP maintenance in vivo. Neuron 38:447-460.

Fukunaga K, Stoppini L, Miyamoto E, Muller D (1993) Long-term potentiation is associated with an increased activity of $\mathrm{Ca}^{2+} /$ calmodulindependent protein kinase II. J Biol Chem 268:7863-7867.

Gardoni F, Schrama LH, van Dalen JJ, Gispen WH, Cattabeni F, Di Luca M (1999) AlphaCaMKII binding to the C-terminal tail of NMDA receptor subunit NR2A and its modulation by autophosphorylation. FEBS Lett 456:394-398.

Giese KP, Fedorov NB, Filipkowski RK, Silva AJ (1998) Autophosphorylation at Thr286 of the alpha calcium-calmodulin kinase II in LTP and learning. Science 279:870-873.

Gleason MR, Higashijima SI, Dallman J, Liu K, Mandel G, Fetcho JR (2003) Translocation of CaM kinase II to synaptic sites in vivo. Nat Neurosci 6:217-218.

Hayashi Y, Shi SH, Esteban JA, Piccini A, Poncer JC, Malinow R (2000) Driving AMPA receptors into synapses by LTP and CaMKII: requirement for GluR1 and PDZ domain interaction. Science 287:2262-2267.

Hudmon A, Schulman H (2002) Structure-function of the multifunctional $\mathrm{Ca}^{2+} /$ calmodulin-dependent protein kinase II. Biochem J 364:593-611.

Illario M, Cavallo AL, Bayer KU, Di Matola T, Fenzi G, Rossi G, Vitale M (2003) Calcium/calmodulin-dependent protein kinase II binds to Raf- 1 and modulates integrin-stimulated ERK activation. J Biol Chem 278:45101-45108.

Kennedy MB (1998) Signal transduction molecules at the glutamatergic postsynaptic membrane. Brain Res Brain Res Rev 26:243-257.

Kim SA, Heinze KG, Waxham MN, Schwille P (2004) Intracellular calmodulin availability accessed with two-photon cross-correlation. Proc Natl Acad Sci USA 101:105-110.

Krapivinsky G, Medina I, Krapivinsky L, Gapon S, Clapham D (2004) The SynGAP-MUPP1-CaMKII synaptic complexes regulate p38 MAP kinase activity and NMDA receptor-dependent synaptic AMPA receptor potentiation. Neuron 43:563-574.

Lee HK, Takamiya K, Han JS, Man H, Kim CH, Rumbaugh G, Yu S, Ding L, He C, Petralia RS, Wenthold RJ, Gallagher M, Huganir RL (2003) Phosphorylation of the AMPA receptor GluR1 subunit is required for synaptic plasticity and retention of spatial memory. Cell 112:631-643.

Leonard AS, Lim IA, Hemsworth DE, Horne MC, Hell JW (1999) Calcium/ calmodulin-dependent protein kinase II is associated with the N-methylD-aspartate receptor. Proc Natl Acad Sci USA 96:3239-3244.

Leonard AS, Bayer KU, Merrill MA, Lim IA, Shea MA, Schulman H, Hell JW (2002) Regulation of calcium/calmodulin-dependent protein kinase II docking to $\mathrm{N}$-methyl-D-aspartate receptors by calcium/calmodulin and alpha-actinin. J Biol Chem 277:48441-48448.

Liao D, Jones A, Malinow R (1992) Direct measurement of quantal changes underlying long-term potentiation in CA1 hippocampus. Neuron 9:1089-1097.

Lisman JE, Zhabotinsky AM (2001) A model of synaptic memory: a CaMKII/PP1 switch that potentiates transmission by organizing an AMPA receptor anchoring assembly. Neuron 31:191-201.

Lisman J, Schulman H, Cline H (2002) The molecular basis of CaMKII function in synaptic and behavioral memory. Nat Rev Neurosci 3:175-190.

Lo DC, McAllister AK, Katz LC (1994) Neuronal transfection in brain slices using particle-mediated gene transfer. Neuron 13:1263-1268. 
Luscher C, Nicoll RA, Malenka RC, Muller D (2000) Synaptic plasticity and dynamic modulation of the postsynaptic membrane. Nat Neurosci 3:545-550.

Makhinson M, Chotiner JK, Watson JB, O’Dell TJ (1999) Adenylyl cyclase activation modulates activity-dependent changes in synaptic strength and $\mathrm{Ca}^{2+} /$ calmodulin-dependent kinase II autophosphorylation. J Neurosci 19:2500-2510.

Malinow R, Schulman H, Tsien RW (1989) Inhibition of postsynaptic PKC or CaMKII blocks induction but not expression of LTP. Science 245:862-866.

Matsuzaki M, Ellis-Davies GC, Kasai H (2003) Structure-function plasticity of single spines of hippocampal CA1 pyramidal neurons induced by twophoton uncaging of a caged-glutamate compound. Soc Neurosci Abstr 29:476.4.

Matsuzaki M, Honkura N, Ellis-Davies GC, Kasai H (2004) Structural basis of long-term potentiation in single dendritic spines. Nature 429:761-766.

Nusser Z, Lujan R, Laube G, Roberts JD, Molnar E, Somogyi P (1998) Cell type and pathway dependence of synaptic AMPA receptor number and variability in the hippocampus. Neuron 21:545-559.

Otmakhov N, Griffith LC, Lisman JE (1997) Postsynaptic inhibitors of calcium/calmodulin-dependent protein kinase type II block induction but not maintenance of pairing-induced long-term potentiation. J Neurosci 17:5357-5365.

Otmakhov N, Carpenter S, Conti R, Lisman J (2001) Measuring CaMKII binding affinity in synaptic spines of living neurons. Soc Neurosci Abstr $27: 610.16$

Otmakhov N, Khibnik L, Otmakhova N, Carpenter S, Riahi S, Asrican B, Lisman J (2004) Forskolin-induced LTP in the CA1 hippocampal region is NMDA receptor dependent. J Neurophysiol 91:1955-1962.

Ouyang Y, Rosenstein A, Kreiman G, Schuman EM, Kennedy MB (1999) Tetanic stimulation leads to increased accumulation of $\mathrm{Ca}^{(2+)}$ / calmodulin-dependent protein kinase II via dendritic protein synthesis in hippocampal neurons. J Neurosci 19:7823-7833.

Piccini A, Malinow R (2002) Critical postsynaptic density 95/disc large/ zonula occludens-1 interactions by glutamate receptor 1 (GluR1) and GluR2 required at different subcellular sites. J Neurosci 22:5387-5392.

Piccini A, Kopec C, Malinow R (2003) Simultaneous measurement of changes in structure and AMPA-r distribution following LTP- and LTDinducing stimuli. Soc Neurosci Abstr 29:375.3.

Shen K, Meyer T (1998) In vivo and in vitro characterization of the sequence requirement for oligomer formation of $\mathrm{Ca}^{2+} /$ calmodulin-dependent protein kinase IIalpha. J Neurochem 70:96-104.

Shen K, Meyer T (1999) Dynamic control of CaMKII translocation and localization in hippocampal neurons by NMDA receptor stimulation. Science 284:162-166.

Shen K, Teruel MN, Subramanian K, Meyer T (1998) CaMKIIbeta functions as an F-actin targeting module that localizes CaMKIIalpha/beta heterooligomers to dendritic spines. Neuron 21:593-606.

Shen K, Teruel MN, Connor JH, Shenolikar S, Meyer T (2000) Molecular memory by reversible translocation of calcium/calmodulin-dependent protein kinase II. Nat Neurosci 3:881-886.

Shi S, Hayashi Y, Esteban JA, Malinow R (2001) Subunit-specific rules governing AMPA receptor trafficking to synapses in hippocampal pyramidal neurons. Cell 105:331-343.

Silva AJ, Stevens CF, Tonegawa S, Wang Y (1992) Deficient hippocampal long-term potentiation in alpha-calcium-calmodulin kinase II mutant mice. Science 257:201-206.

Strack S, Colbran RJ (1998) Autophosphorylation-dependent targeting of calcium/calmodulin-dependent protein kinase II by the NR2B subunit of the $N$-methyl-D-aspartate receptor. J Biol Chem 273:20689-20692.

Strack S, Choi S, Lovinger DM, Colbran RJ (1997) Translocation of autophosphorylated calcium/calmodulin-dependent protein kinase II to the postsynaptic density. J Biol Chem 272:13467-13470.

Strack S, McNeill RB, Colbran RJ (2000a) Mechanism and regulation of calcium/calmodulin-dependent protein kinase II targeting to the NR2B subunit of the $\mathrm{N}$-methyl-D-aspartate receptor. J Biol Chem 275:23798-23806.

Strack S, Robison AJ, Bass MA, Colbran RJ (2000b) Association of calcium/ calmodulin-dependent kinase II with developmentally regulated splice variants of the postsynaptic density protein densin-180. J Biol Chem 275:25061-25064.

Svoboda K, Tank DW, Denk W (1996) Direct measurement of coupling between dendritic spines and shafts. Science 272:716-719.

Walikonis R, Oguni A, Khorosheva E, Jeng C, Asuncion F, Kennedy M (2001) Densin-180 forms a ternary complex with the $(\alpha)$-subunit of $\mathrm{Ca}^{2+} /$ calmodulin-dependent protein kinase II and $(\alpha)$-actinin. J Neurosci 21:423-433.

Yuste R, Majewska A, Holthoff K (2000) From form to function: calcium compartmentalization in dendritic spines. Nat Neurosci 3:653-659. 